\title{
Setting safety stocks in multi-stage inventory systems under rolling horizon mathematical programming models
}

\author{
Youssef Boulaksil • Jan C. Fransoo • \\ Ernico N. G. van Halm
}

Published online: 12 May 2007

(C) Springer-Verlag 2007

\begin{abstract}
This paper considers the problem of determining safety stocks in multi-item multi-stage inventory systems that face demand uncertainties. Safety stocks are necessary to make the supply chain, which is driven by forecasts of customer orders, responsive to (demand) uncertainties and to achieve predefined target service levels. Although there exists a large body of literature on determining safety stock levels, this literature does not provide an effective methodology that can address complex multi-constrained supply chains. In this paper, the problem of determining safety stocks is addressed by a simulation based approach, where the simulation studies are based on solving the supply chain planning problem (formulated as a mathematical programming model) in a rolling horizon setting. To demonstrate the utility of the proposed approach, an application of the approach at Organon, a worldwide operating biopharmaceutical company, will be discussed.
\end{abstract}

Keywords Safety stocks $\cdot$ Advanced planning and scheduling $\cdot$ Simulation $\cdot$ Supply chain planning $\cdot$ Organon

\section{Introduction}

Supply chains are exposed to different types of uncertainties that stem from random yields, processing times or forecast errors. These uncertainties can be covered to a

\footnotetext{
Y. Boulaksil $(\bowtie) \cdot$ J. C. Fransoo

Department of Technology Management, Technische Universiteit Eindhoven,

Eindhoven, The Netherlands

e-mail: Y.Boulaksil@tue.nl

J. C. Fransoo

e-mail: J.C.Fransoo@tue.nl

E. N. G. van Halm

Supply Chain Management Department, Organon N.V., Oss, The Netherlands

e-mail: ernico.vanhalm@organon.com
} 
large extent by mechanisms like safety time, safety stocks or combinations of these (Whybark and Williams 1976; Wijngaard and Wortmann 1985). This paper focuses on the determination of safety stocks in multi-item multi-stage inventory systems that face demand uncertainties. We assume that the inventory system is planned and controlled by a central decision authority that plans the supply chain based on deterministic mathematical programming models. However, demand uncertainty is an important factor to be considered in supply chain planning. Planning systems based on mathematical programming models are widely implemented in so-called Advanced Planning and Scheduling systems (APS) (Stadtler and Kilger 2005).

When a particular supply chain is facing demand uncertainties, stock outs can occur at all stages in the supply chain. A stock out may cause lost sales, emergency shipments, or loss of goodwill. Therefore, safety stocks should be kept to increase the service levels. Traditionally, safety stocks are determined in advance based on models from inventory theory (Silver et al. 1998). However, it is not obvious how to determine safety stock levels that cover demand uncertainties in complex supply chains that face several constraints such as batch sizes, capacity constraints, non-stationary demand process or forecast errors.

The approach proposed in this paper enables the determination of safety stocks in multi-item multi-stage inventory systems that face demand uncertainties. This approach considers all kinds of constraints that are also considered in supply chain planning practice such as batch sizes and capacity and materials constraints. The approach is based on a simulation of the supply chain planning model in a rolling horizon setting. Based on target service levels, safety stocks are determined after performing simulations, assuming that the demand process and replenishment decisions are independent of the safety stock levels.

The core of the approach is solving the supply chain planning problem very frequently, where the safety stocks are excluded from the supply chain planning model or by setting them equal to zero. Since we assume that all unsatisfied demand is backordered at all stages in the supply chain, backorder quantities are recorded after each solving round. The safety stock level is an increasing function of the target service level, which we measure by the fill rate, i.e. the long-run fraction of demand satisfied routinely from the shelf (Silver et al. 1998). Based on the stored backorder quantities and the target service levels, safety stock levels can be determined.

The proposed approach is suitable for companies that have implemented an APS. APS systems are planning systems that are based on cost minimization models that ensure that, given the resource and material availability constraints of the production system and given certain service level constraints, the best possible quantity of a certain item is released at the lowest value of the objective function. These planning systems are based on mathematical programming models that are solved in a rolling horizon setting (Spitter et al. 2005).

The proposed approach has been applied successfully at Organon, a worldwide operating biopharmaceutical company with production sites, warehouses, and distribution centers spread all over the world.

The remainder of this paper is organized as follows. Section 2 discusses a literature review on this topic. Next, Sect. 3 discusses the problem definition and thereafter, Sect. 4 discusses the proposed approach. The approach has been applied in a real-life 
situation, which is discussed in Sect. 5. Finally, Sect. 6 draws some conclusions about the approach.

\section{Literature review}

There is an extensive amount of literature available on inventory control models in multi-stage or multi-echelon inventory systems incorporating uncertainties. We refer the reader to survey articles by Van Houtum et al. (1996) and Diks et al. (1996). Our research is within the field of Supply Chain Operations Planning (De Kok and Fransoo 2003). The objective of Supply Chain Operations Planning is to coordinate the release of materials and resources in a supply chain network such that customer service constraints are met at minimal costs (De Kok and Fransoo 2003). Two different approaches exist for modelling the Supply Chain Operations Planning problem.

One approach is based on multi-echelon stochastic inventory theory. In this approach, demand that is faced by the supply chain is modelled as a stochastic variable. The key decisions of this approach are the inventory positioning at the various stockpoints in the supply chain, the allocation of quantities at inventory points where the product flow diverges, and the determination of safety stock levels at the several stockpoints. Therefore, the determination of safety stocks is defined as part of the problem. Lead times are (deterministic) input variables to the model and capacity is assumed to be controlled through a combination of order acceptance in the demand management function and a workload control function in the production department. The logic is based on a line of research that has been initiated by Clark and Scarf (1960).

The alternative approach is based on mathematical programming principles. In this approach, demand is inserted into the model as forecasts for every period in the planning horizon. Safety stocks are input parameters to the model and the key decisions are the allocation of inventory quantities at the stockpoints in the supply chain. Lead times are either modelled as deterministic input variables (e.g., Spitter et al. 2005) or are observed as output variables of the model (e.g., Stadtler 2003). Capacity constraints are modelled explicitly as aggregate constraints. The principles are based on research stemming from advanced MRP modelling (Billington et al. 1983) or from multi-period lot sizing problems (Tempelmeier and Derstroff 1996; Stadtler 2003). The principles have been implemented in commercial software, mostly using CPLEX solving logic (See also Stadtler and Kilger 2005).

The two approaches differ also from a safety stock perspective. In this first approach, safety stocks are defined as part of the problem, whereas in the second approach safety stocks are input parameters to the planning model, which have to be determined externally.

This paper focuses on the determination of safety stocks for the latter type of planning approaches. A lot of papers appeared in the last decades on determining safety stocks in multi-stage or multi-echelon inventory models for covering demand uncertainties. We mention a set of papers that are related to our work.

Inderfurth and Minner (1998) propose a dynamic programming approach to treat the problem of determining safety stocks in multi-stage inventory systems, assuming normally distributed demand and periodic review base stock control policies. 
Furthermore, they also assume that no internal delays occur and that each stockpoint is satisfying a service level constraint. More approaches that are based on dynamic programming algorithms can be found in Inderfurth (1991) and Minner (1997).

Graves and Willems (2000) discuss the so-called guaranteed-service model for setting safety stocks in a multi-stage setting to cover demand uncertainties. They develop a model for positioning safety stocks in a supply chain where each stage is controlled by a base-stock policy, assuming an upper bound for the (customer) demand level. Therefore, the safety stocks set by their approach cover demand realizations below the upper bounds. This assumption is necessary to model guaranteed service times between each stage in the supply chain and its customers.

There are also papers on determining safety stocks in multi-stage inventory systems where the approach is based on simulation studies. Optimization methodologies based on simulation of inventory systems are discussed in Kleijnen and Wan (2006).

Eilon and Elmaleh (1968) perform simulation studies to compare the performance of five alternative inventory control policies given wide fluctuating and seasonal demand patterns. The results of the simulations are several non-linear curves showing the relation between the fill rate and mean stock level. Three of these five control policies include safety stocks, but the authors do not discuss how they determined the safety stock parameters.

Wemmerlöv and Whybark (1984) also perform simulation experiments to evaluate several single-stage lot sizing procedures under demand uncertainty. Cost comparisons of the procedures are made with a service level of at least $99.999 \%$. The safety stocks needed to achieve these service levels are determined by a search routine, i.e. repeating the simulations until the target service levels are reached.

De Bodt and Van Wassenhove (1983) present a case study at a company, which uses MRP in a dynamic environment, i.e. the company faces substantial demand uncertainties. The safety stock setting is analysed by a simulation study. Several strategies were defined (combinations of safety stock and safety time) and analysed which resulted in graphs relating average inventory level to service levels. They provide managerial insight by showing that considerable savings can be made at this company, but do not discuss how the safety stocks should be determined.

In the studies of Callarman and Hamrin (1984) the performance of three lot sizing rules in MRP systems is compared, given an uncertain demand process. The cost comparisons have been made by introducing safety stocks at each run to keep the service levels at 95 and $98 \%$. The required safety stocks are determined by using the so-called Service Level Decision Rule (SLDR), which has been developed by Callarman and Mabert (1978). The SLDR is based on linear regression analysis on simulated values of the following set of factors: forecast errors, coefficient of variation of demand, and the expected time between orders. In order to achieve the target service level, the SLDR is used with a search routine.

Our work is closely related to Kohler-Gudum and De Kok (2001) who propose a so-called Safety Stock Adjustment Procedure (SSAP) to obtain target service levels in simulation models. The technique is based on the assumption that a Time Phased Order Point (TPOP) policy is applied. Their simulation study aims to determine the discrete probability density function of the net stock process. Based on this probability distribution, the safety stock is adjusted to ensure the specified target service level. 
Our approach differs from Kohler-Gudum and De Kok (2001) on two aspects. First, our approach determines the empirical distribution of the backorder quantities for setting safety stocks instead of determining the probability density function of the inventory process. Based on a set of assumptions (independence of the demand and replenishment process of safety stock levels), the necessary amount of safety stock is determined by adding the adjustment quantity to the initial safety stock that can be an arbitrary value. In our approach, the initial safety stock is set equal to zero and afterwards, the safety stock level is determined based on backordered quantities. Second, the model that is used in our approach is a planning model that is solved in rolling horizon setting, and therefore, the planning process is imitated as much as possible. Kohler-Gudum and De Kok (2001) do not discuss the simulation model extensively.

Although there exists a large body of literature on determining safety stock levels, to our knowledge, this literature does not provide an effective methodology that can address supply chains that face several constraints like capacity constraints, production in batch sizes, and non-stationary forecast process. Most approaches make restrictive assumptions about the demand process (Inderfurth and Minner 1998; Graves and Willems 2000) or do not explicitly discuss how they set the safety stock levels (Eilon and Elmaleh 1968; De Bodt and Van Wassenhove 1983). Our approach is closely related to Kohler-Gudum and De Kok (2001), but we extend the approach by using an empirical supply chain planning model in the simulation study and that makes the results of the approach more reflecting the (planning) practice.

\section{Problem definition}

We consider a supply chain that consists of an arbitrary number of stages and stockpoints in which a product passes through multiple production sites before it is finally delivered to outside customers. This supply chain is planned and controlled by a central decision authority that has access to all relevant status information (like inventory levels and work-in-process quantities) at all production sites and makes release decisions for the entire supply chain. The release decisions result from a deterministic mathematical programming model that is solved in a rolling horizon setting (Stadtler and Kilger 2005), which has been implemented in an Advanced Planning and Scheduling system. For these kinds of planning models, safety stocks are input parameters that have to be determined externally.

Formulation of the planning problem by a mathematical programming model assumes a deterministic view of supply chain planning by considering all model parameters, as demand, lead times, production rates to be known with complete certainty. This assumption of complete and deterministic information is desirable from a model complexity point of view, but given the dynamic and uncertain nature of most supply chains, this assumption is violating reality. Demand uncertainty is an important factor to be considered in supply chain planning, and therefore, safety stocks are kept to cover part of the demand uncertainties.

The core function of supply chain planning models is to coordinate material and resource release decisions in the supply chain such that predefined customer service levels are achieved with minimal costs. Safety stocks are kept to deal with 
demand uncertainties and consequently to increase service levels. The service level is an increasing function of the safety stock level. Therefore, more safety stocks are needed to increase the service level, which results in increased inventory holding costs.

From the other side, demand uncertainties can cause stock outs that result in lost sales, emergency shipments, or loss of goodwill. Since we assume that all excess demand that is not directly satisfied from inventory is backordered, costs that are related with a backorder are backorder costs, which are harder to quantify than inventory holding costs. The problem of setting safety stocks is mainly a trade-off between inventory holding costs and backorder costs. Section 4 discusses the modelling of these costs and discusses also the considered supply chain planning model in detail.

\section{The approach}

We consider a supply chain that is planned and controlled by a central decision authority, which may be supported by an Advanced Planning and Scheduling system. We assume that the supply chain planning model is based on a mathematical programming model that is solved in a rolling horizon setting, where the forecasts may be updated when the planning horizon is shifted. We also assume that the demand process and replenishment decisions are independent of the safety stock level. Furthermore, we assume that all excess demand at all stages in the supply chain is backordered. We do not make any assumption about the demand and forecast process, which makes this approach less restrictive to a certain probability density function of the demand process.

Based on the discussed assumptions, a simulation experiment is performed in the following way. The planning horizon is divided into a fixed number of time buckets, which are filled by forecasts of the demand generated by a demand generator, which generates a series of forecasts based on historical demand and forecasts data. Then, the planning model with demand forecasts is solved given all kinds of materials and resources constraints. The planning model may be based on linear programming models or mixed-integer programming models if some decisions require integer variables. Such discrete decisions can, among others, regard lotsizing in production or transportation. At the end of the first time bucket (planning cycle), the state of the system (e.g. the inventory levels and forecasts) is updated and the planning cycle is repeated with the horizon shifted by one period.

Figure 1a illustrates the inventory development of a certain product and the resulting backorder process that is output of 100 simulation runs. After the simulation runs, the horizontal axis is shifted (see Fig. 1b) such that the number of backorders is limited, i.e. the customer service level is increased to a certain predefined level. Figure $1 \mathrm{~b}$ shows that increasing the safety stock level decreases the number of backorders, and therefore, increases the service level.

Thus, by solving the supply chain planning problem very frequently where each time the forecasts are updated, long-run backorder quantities indicate the amount of safety stocks that was needed to prevent the backorders partially, i.e. to achieve a certain customer service level. Note that the customer service level is externally determined for all products at all stages in the supply chain. The customer service level has 

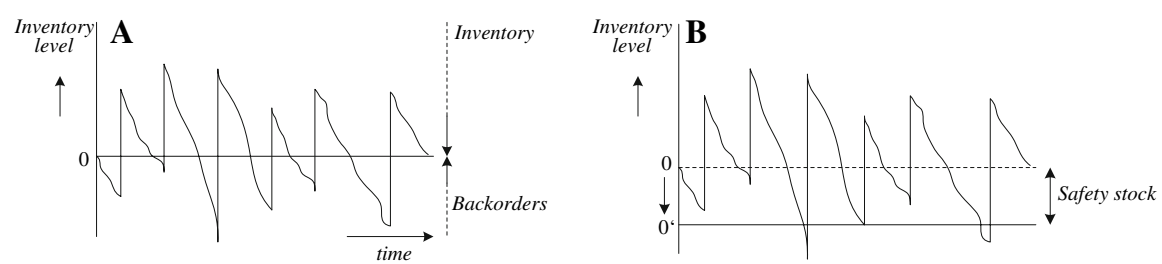

Fig. 1 The inventory development of a certain product; a shows the results of simulation runs and $\mathbf{b}$ shows how the horizontal axis is shifted to limit the number of backorders, i.e. to achieve a certain customer service level

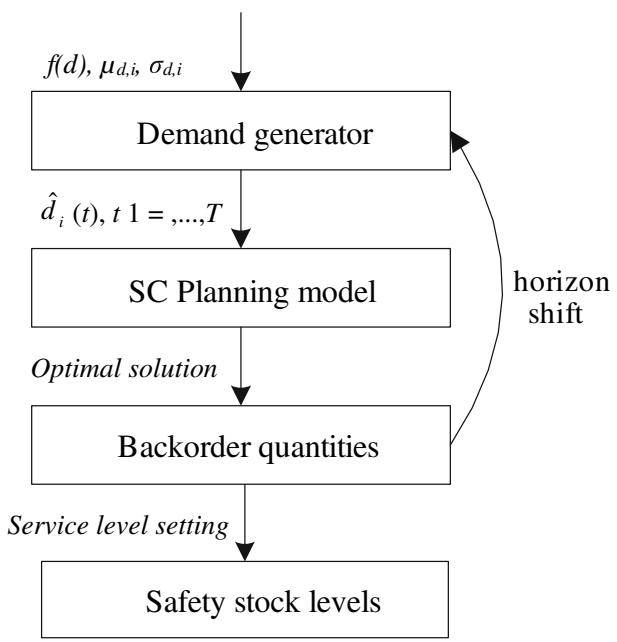

Explanation of used symbols

$f(d) \quad$ probability density function of the demand process

$\mu_{d, i} \quad$ Expected (exogenous) demand of end-item $i$

$\sigma_{d, i} \quad$ Standard deviation of forecast errors of end-iem $i$

$T \quad$ Planning horizon

$\hat{d}_{i}(t) \quad$ Forecast of demand of end-item $i$ in period $t(t=1, \ldots, T)$

Fig. 2 Several steps of the approach

been defined as the long-run fraction of demand satisfied directly from shelf (fill rate measure). Having discussed the theoretical idea behind the approach, the steps of the approach (see Fig. 2) will be discussed in the following sections in more detail.

\subsection{Demand generator}

The first step of the approach is the generation of a series of forecasts which are input to the supply chain planning model. We do not make any assumption about the distribution of the demand process. Historical data about demand and forecasts may be (statistically) fitted into the best fitting probability distribution function. Having chosen the most suitable probability density function for the demand distribution, the 
first two moments of the distribution can be derived to determine the parameters of the demand distribution. Figure 2 shows that these parameters $\left(\mu_{d, i}, \sigma_{d, i}\right)$ are input for the demand generator where $\mu_{d, i}$ is the expected (exogenous) demand of end-item $i$ and $\sigma_{d, i}$ is the standard deviation of forecast errors of end-item $i$.

Suppose that we have historical sales data of $n$ time periods, then $\mu_{d, i}$ can be calculated by

$$
\mu_{d, i}=\frac{1}{n} \sum_{s=-n}^{-1} d_{i}(t+s)
$$

where $d_{i}(t)$ is the demand for item $i$ in period $t$. Forecast errors can be determined by several measures (Silver et al. 1998). The Mean Absolute Deviation (MAD) is recommended for its computational simplicity. The MAD for item $i$ as function of the forecast horizon $h$ can be calculated by

$$
\operatorname{MAD}_{i}(h)=\frac{1}{T} \sum_{s=-T}^{-1}\left(d_{i}(t+s)-\hat{d}_{i}(t+s-h, t+s)\right)
$$

where $T$ is the length of the planning horizon, $d_{i}(t)$ the demand for item $i$ in period $t$, and $\hat{d}_{i}(t-h, t)$ the forecast made in period $t-h$ for the demand in period $t$. It is reasonable to assume that the MAD is an increasing function of the forecast horizon $h$ (Heath and Jackson 1994). The conversion of $\mathrm{MAD}_{i}(h)$ to $\sigma_{i}(h)$ is extensively discussed in Silver et al. (1998). Having determined the parameters of the demand distribution, the random generator can generate a series of forecasts of the demand $\hat{d}_{i}(t)$ for $t=1, \ldots, T$. The generated forecasts are input for the supply chain planning model.

Several extensions are possible. For example, the demand process may not be stationary which makes the $\mu_{d, i}$ a function of time (demand process follows a trend or has a seasonal effect). Furthermore, the standard deviation of the forecast errors may be not a function of the forecast horizon $h$. Several kinds of adaptations are possible in order to imitate the demand and forecast process as much as possible.

\subsection{Supply chain planning model}

One approach to supply chain planning models is based on deterministic mathematical programming principles (De Kok and Fransoo 2003). The advantage of using the supply chain planning model (implemented in an APS system) for the simulation study is that it already contains the network structure(s), the item list, bill-of-materials structure, batch sizes, and the routings. Using the supply chain planning model for this approach is highly recommended, as these models are reflecting the planning practice. Furthermore, for those companies that have implemented an APS system, little modelling effort is required for this approach.

The mathematical programming model that is used to determine the safety stock levels is a stand-alone model, but derived from the supply chain planning model. The supply chain planning model may have to be adapted, as safety stocks have to be set 


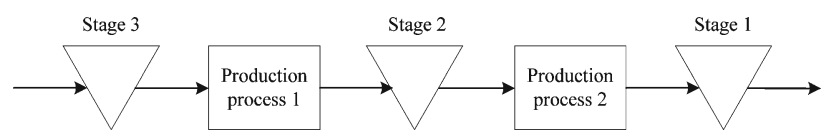

Fig. 3 A three-stage supply chain considered in the supply chain planning model

equal to zero in the supply chain planning models or replenishment decisions should not consider safety stock levels. Then, the planning problem should be solved without considering safety stocks, i.e. backorders are planned when demand exceeds available inventories. Depending on the supply chain planning model, the planning problem has to be solved such that all relevant cost factors have to be considered except costs associated with consumption of safety stocks. The solution of the supply chain planning model contains order releases for the production system and planned inventory levels. The order releases within the length of the lead time of a certain item at a certain stage (frozen horizon) are stored, as they are not allowed to be changed in the next solving round. Below, we discuss the considered supply chain planning model in detail, which is used to determine the safety stock levels. Figure 3 shows a rough outline of the three-stage supply chain that is considered in the supply chain planning model.

\subsubsection{Objective function}

Equation (3) is the objective functions of the supply chain planning model. The objective function minimizes the total costs (TC), which consist of several cost factors that are assigned to several stages in the supply chain. We consider a (pharmaceutical) supply chain with three stages. Stage 3 is the most upstream stage where the raw materials (active ingredients) are stored. The active ingredients are processed to tablets, which are stored at the second stage. Thereafter, the tablets are packaged and stored at the most downstream stage (stage 1 ). $N_{j}$ is the total number of items at stage $j$ with $j \in\{1,2,3\}, n_{j}$ is a certain item that belongs to stage $j, t$ is a certain (discrete) time period, and $T$ is the planning horizon.

$$
\begin{aligned}
\operatorname{Min} \mathrm{TC}= & \sum_{n_{1}} \sum_{t=1}^{T} c_{1} \cdot \mathrm{UD}_{n_{1}}(t)+\sum_{n_{1}} \sum_{t=1}^{T} c_{2} \cdot \mathrm{OP}_{n_{1}} \cdot \mathrm{BM}_{n_{1}}(t) \\
& +\sum_{n_{1}} \sum_{t=1}^{T} c_{3} \cdot \mathrm{EI}_{n_{1}}(t)+\sum_{n_{2}} \sum_{t=1}^{T} c_{4} \cdot \mathrm{UD}_{n_{2}}(t) \\
& +\sum_{n_{2}} \sum_{t=1}^{T} c_{5} \cdot \mathrm{UDD}_{n_{2}}(t)+\sum_{n_{2}} \sum_{t=1}^{T} c_{6} \cdot \mathrm{BC}_{n_{2}}(t) \\
& +\sum_{n_{2}} \sum_{t=1}^{T} c_{7} \cdot \mathrm{EI}_{n_{2}}(t)+\sum_{n_{3}} \sum_{t=1}^{T} c_{8} \cdot \mathrm{UD}_{n_{3}}(t) \\
& +\sum_{n_{3}} \sum_{t=1}^{T} c_{9} \cdot \mathrm{UDD}_{n_{3}}(t)+\sum_{n_{3}} \sum_{t=1}^{T} c_{10} \cdot \mathrm{EI}_{n_{3}}(t)
\end{aligned}
$$


The first three terms of the objective function are related to the first stage in the supply chain. For this stage, we consider three cost factors that have to be minimized. The first terms are costs associated with unsatisfied demand (backorders) $c_{1} \cdot \mathrm{UD}_{n_{1}}(t)$ where $\mathrm{UD}_{n_{1}}(t)$ is the backorder quantity for item $n_{1}$ in period $t$. The second term deals with costs for replenishing a quantity that deviates from the (minimum) replenishment quantity $c_{2} \cdot \mathrm{OP}_{n_{1}} \cdot \mathrm{BM}_{n_{1}}(t) \cdot \mathrm{OP}_{n_{1}}$ is the period order quantity for item $n_{1}$ and $\mathrm{BM}_{n_{1}}(t)$ the deviation from the minimum replenishment quantity for item $n_{1}$ in period $t$. Campaign sizes are determined based on a trade-off between ordering costs and inventory holding costs, whereas batch sizes are quantities that are determined by legislative authorities. Therefore, producing in fixed batch sizes is required, whereas deviating from the campaign size is undesired. The third term is the total inventory holding cost at this stage $c_{3} \cdot \mathrm{EI}_{n_{1}}(t)$ where $\mathrm{EI}_{n_{1}}(t)$ is the inventory level of item $n_{1}$ at the end of period $t$.

For the second stage of the supply chain, four cost factors are considered. The first terms sum backorders that result from exogenous demand at this stage. So, $c_{4} \cdot \mathrm{UD}_{n_{2}}(t)$ is unsatisfied demand (backorder) costs for item $n_{2}$ in time period $t$, whereas the second term $c_{5} \cdot \mathrm{UDD}_{n_{2}}(t)$ considers unsatisfied demand (backorders) that result from endogenous (derived) demand from the first stage of the supply chain. Since the second stage in the supply chain considers the production of tablets in campaigns (a fixed multiple of batch sizes), the third term $c_{6} \cdot \mathrm{BC}_{n_{2}}(t)$ considers costs associated with deviating from the fixed campaign size $\mathrm{BC}_{n_{2}}(t)$ for item $n_{2}$ in period $t$. The fourth term considers the total inventory holding costs for item $n_{2}$.

The third stage in the supply chain considers three cost factors: costs associated with unsatisfied demand (backorders) of exogenous demand $c_{8} \cdot \operatorname{UD}_{n_{3}}(t)$, costs associated with unsatisfied demand (backorders) that result from endogenous demand (from the second stage of the supply chain) $c_{9} \cdot \operatorname{UDD}_{n_{3}}(t)$, and total inventory holding costs $c_{10} \cdot E I_{n_{3}}(t)$ of item $n_{3}$. For confidentially reasons, we cannot show the values of the cost parameters, except that $c_{1}>c_{2}>\cdots>c_{10}$. The determination of these cost parameters was not part of this study, as they can be taken over from the objective function of the supply chain planning model.

\subsubsection{Stage 1 model}

The objective function (3) is minimized subject to several constraints, which are discussed below per stage in the supply chain. Equations (4) are materials balance equations with $\mathrm{EI}_{n_{1}}(t)$ is the inventory level of item $n_{1}$ at the end of period $t, T R_{n_{1}}(t)$ the replenishment quantity of item $n_{1}$ in period $t$, and $\operatorname{TD}_{n_{1}}(t)$ the (exogenous) demand for item $n_{1}$ in period $t$. The latter parameter contains data that are input to the planning model. Further, $\mathrm{EI}_{n_{1}}(0)$ is the initial inventory level.

$$
\mathrm{EI}_{n_{1}}(t)=\mathrm{EI}_{n_{1}}(t-1)+\mathrm{TR}_{n_{1}}(t)-\mathrm{TD}_{n_{1}}(t), \quad n_{1}=1, \ldots, N_{1}, t=1, \ldots, T
$$

Equation (5) determine the minimum replenishment quantity for item $n_{1}$ in period $t$, as the replenishments are based on periodic order quantity (OP). $I D_{n_{1}}(t)$ is the (forecast of) independent demand for item $n_{1}$ in period $t$. 


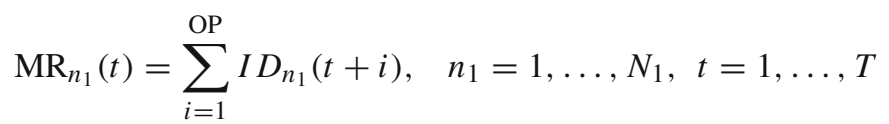

Having determined the minimum replenishment quantity, Eq. (6) determine the real replenishment quantities $\mathrm{SM}_{n_{1}}(t)$ for item $n_{1}$ in period $t . \mathrm{BM}_{n_{1}}(t)$ is then the deviation from minimum replenishment quantity for item $n_{1}$ in period $t$ that is considered in the objective function.

$$
\operatorname{SM}_{n_{1}}(t)=\mathrm{MR}_{n_{1}}(t)-\mathrm{BM}_{n_{1}}(t), \quad n_{1}=1, \ldots, N_{1}, t=1, \ldots, T
$$

The total replenishment quantity for item $n_{1}$ for the entire planning horizon $\left(\mathrm{TR}_{n_{1}}\right.$ $(t))$ is determined by two parts: $\operatorname{SM}_{n_{1}}(t)$ which we have just discussed and $\operatorname{FP}_{n_{1}}(t)$ which are fixed replenishment quantities of item $n_{1}$ in period $t$ determined in previous solving rounds. The binary parameter $\alpha$ regulates that within the lead time of the planning horizon no new decisions are taken.

$$
\begin{aligned}
& \operatorname{TR}_{n_{1}}(t)=\alpha \cdot S M_{n_{1}}(t)+(1-\alpha) \cdot \mathrm{FP}_{n_{1}}(t), \quad n_{1}=1, \ldots, N_{1}, \\
& t=1, \ldots, T, \alpha= \begin{cases}0 & \text { if } t \leqslant L \\
1 & \text { if } L<t \leqslant T\end{cases}
\end{aligned}
$$

Equation (8) determine which part of the exogenous demand $\operatorname{ID}_{n_{1}}(t)$ for item $n_{1}$ in period $t$ is satisfied $\left(\operatorname{SD}_{n_{1}}(t)\right)$. The unsatisfied demand quantity $\operatorname{UD}_{n_{1}}(t)$ for item $n_{1}$ in period $t$ is punished in the objective function.

$$
\mathrm{SD}_{n_{1}}(t)=\mathrm{ID}_{n_{1}}(t)-\mathrm{UD}_{n_{1}}(t), \quad n_{1}=1, \ldots, N_{1}, t=1, \ldots, T
$$

Equation (9) are equations for $\operatorname{TD}_{n_{1}}(t)$ which is determined by $\operatorname{SD}_{n_{1}}(t)$ that result from Eq. (8) plus $\mathrm{UD}_{n_{1}}(t-1)$ which is the unsatisfied demand in $t-1$, i.e. backorder quantity for item $n_{1}$ from period $t$.

$$
\mathrm{TD}_{n_{1}}(t)=\mathrm{SD}_{n_{1}}(t)+\mathrm{UD}_{n_{1}}(t-1), \quad n_{1}=1, \ldots, N_{1}, t=1, \ldots, T
$$

\subsubsection{Stage 2 model}

Several constraints apply to stage 2 which will be discussed now. Like in stage 1 , Eq. (10) are the balance equations for the materials flow. The symbols have the same meaning as in stage 1, except that indices show that the equations apply to this particular stage.

$$
\mathrm{EI}_{n_{2}}(t)=\mathrm{EI}_{n_{2}}(t-1)+\mathrm{TR}_{n_{2}}(t)-\mathrm{TD}_{n_{2}}(t), \quad n_{2}=1, \ldots, N_{2}, t=1, \ldots, T
$$

Equation (11) determine the total replenishment quantity for item $n_{2}$ in period $t$ where $\alpha$ is the same binary parameter that is used in Eq. (7). $P P_{n_{2}}(t)$ is the production 
quantity to be produced of item $n_{2}$ in $t$ and $\mathrm{FP}_{n_{2}}(t)$ are firmed production quantities that are determined in previous solving rounds.

$T R_{n_{2}}(t)=\alpha \cdot P P_{n_{2}}(t)+(1-\alpha) \cdot F P_{n_{2}}(t), \quad n_{2}=1, \ldots, N_{2}, t=1, \ldots, T$

Equation (12) require that the production quantity of item $n_{2}$ to be produced in period $t$ must be an integer multiple of $Q_{n_{2}}$, the batch size of item $n_{2}$ multiplied by $y_{n_{2}}$, the yield factor of the production process that produces item $n_{2}$.

$$
\operatorname{PP}_{n_{2}}(t)=Q_{n_{2}} \cdot y_{n_{2}} \cdot \mathrm{NB}_{n_{2}}(t), \quad n_{2}=1, \ldots, N_{2}, t=1, \ldots, T,
$$

with $N B_{n_{2}}(t) \in \mathrm{N}_{0}$.

Equation (13) determine the derived (endogenous) demand at stage 2. This is the multiplication of the (with lead time $L$ shifted) replenishment quantities of items $n_{1}$ with the $\mathrm{BOM}$ factor.

$$
\mathrm{DD}_{n_{2}}(t)=\mathrm{BOM}_{n_{2}, n_{1}} \cdot \sum_{n_{1}} \mathrm{SM}_{n_{1}}(t-L), \quad n_{2}=1, \ldots, N_{2}, t=1, \ldots, T
$$

Equation (14) determine the costs associated with going below the campaign size $\mathrm{BC}_{n_{2}}(t)$, which is punished in the objective function. $\mathrm{CS}_{n_{2}}$ is the campaign size (a certain number of batches of $n_{2}$ ) of item $n_{2}$.

$$
\mathrm{BC}_{n_{2}}(t)=\mathrm{CS}_{n_{2}}-\mathrm{NB}_{n_{2}}(t), \quad n_{2}=1, \ldots, N_{2}, t=1, \ldots, T
$$

with $\mathrm{BC}_{n_{2}}(t) \in \mathrm{N}_{0}$.

Unsatisfied demand from $t-1$ (resulting from either exogenous demand $\mathrm{UD}_{n_{2}}(t-1)$ or endogenous demand $\mathrm{UDD}_{n_{2}}(t-1)$ determine the backorder quantity $\mathrm{BO}_{n_{2}}(t)$ of item $n_{2}$ in period $t$.

$$
\mathrm{BO}_{n_{2}}(t)=\mathrm{UDD}_{n_{2}}(t-1)+\mathrm{UD}_{n_{2}}(t-1), \quad n_{2}=1, \ldots, N_{2}, t=1, \ldots, T
$$

Equation (16) show that the satisfied part of demand for item $n_{2}$ in period $t \operatorname{SD}_{n_{2}}(t)$ is equal to the exogenous demand $\operatorname{ID}_{n_{2}}(t)$ for item $n_{2}$ in period $t$ minus unsatisfied demand quantity $\mathrm{UD}_{n_{2}}(t)$ for item $n_{2}$ in period $t$, which is punished in the objective function.

$$
\mathrm{SD}_{n_{2}}(t)=\mathrm{ID}_{n_{2}}(t)-\mathrm{UD}_{n_{2}}(t), \quad n_{2}=1, \ldots, N_{2}, t=1, \ldots, T
$$

Equation (17) are the application of the same idea (as Eq. 16) to the dependent (endogenous) demand for item $n_{2}$ in period $t$.

$$
\operatorname{SDD}_{n_{2}}(t)=\mathrm{DD}_{n_{2}}(t)-\mathrm{UDD}_{n_{2}}(t), \quad n_{2}=1, \ldots, N_{2}, t=1, \ldots, T
$$


The sum of $\mathrm{SD}_{n_{2}}(t), \mathrm{SDD}_{n_{2}}(t)$, and the backorders for item $n_{2}$ in period $t \mathrm{BO}_{n_{2}}(t)$ are equal to $\operatorname{TD}_{n_{2}}(t)$, total demand for item $n_{2}$ in period $t$.

$$
\mathrm{TD}_{n_{2}}(t)=\mathrm{SDD}_{n_{2}}(t)+\mathrm{SD}_{n_{2}}(t)+\mathrm{BO}_{n_{2}}(t), \quad n_{2}=1, \ldots, N_{2}, t=1, \ldots, T
$$

\subsubsection{Stage 3 model}

Constraints (19) till (26) apply to the third stage of the supply chain. Equation (19) are the balance equations for this stage. $\mathrm{EI}_{n_{3}}(t)$ is the inventory level of item $n_{3}$ at the end of period $t, \operatorname{TR}_{n_{3}}(t)$ is the replenishment quantity of item $n_{3}$ in period $t$, and $\operatorname{TD}_{n_{3}}(t)$ is the total demand of item $n_{3}$ in period $t$.

$$
\mathrm{EI}_{n_{3}}(t)=\mathrm{EI}_{n_{3}}(t-1)+\mathrm{TR}_{n_{3}}(t)-\mathrm{TD}_{n_{3}}(t), \quad n_{3}=1, \ldots, N_{3}, t=1, \ldots, T
$$

The replenishment quantity $\mathrm{TR}_{n_{3}}(t)$ is partly determined in the previous solving rounds $\left(\mathrm{FP}_{n_{3}}(t)\right.$, firm planned replenishment orders for item $n_{3}$ in period $\left.t\right)$ and new released orders $O_{n_{3}}(t)$ to be determined for item $n_{3}$ in period $t$. The orders are sent to (external) supplier(s).

$$
\mathrm{TR}_{n_{3}}(t)=(1-\alpha) \cdot \mathrm{FP}_{n_{3}}(t)+\alpha \cdot O_{n_{3}}(t), \quad n_{3}=1, \ldots, N_{3}, t=1, \ldots, T
$$

Furthermore, constraints (21) require that the ordered items are (a) integer multiple(s) of $Q_{n_{3}}$, batch sizes for item $n_{3}$.

$$
O_{n_{3}}(t)=\mathrm{NB}_{n_{3}}(t) \cdot Q_{n_{3}}, \quad n_{3}=1, \ldots, N_{3}, t=1, \ldots, T,
$$

with $\mathrm{NB}_{n_{3}}(t) \in \mathrm{N}_{0}$.

The total demand for item $n_{3}$ is determined by adding the satisfied parts of the dependent (endogenous), independent (exogenous) demand plus the backorders for item $n_{3}$ in period $t$.

$$
\mathrm{TD}_{n_{3}}(t)=\mathrm{SDD}_{n_{3}}(t)+\mathrm{SD}_{n_{3}}(t)+\mathrm{BO}_{n_{3}}(t), \quad n_{3}=1, \ldots, N_{3}, \quad t=1, \ldots, T
$$

Equations (23) and (24) show how the satisfied parts of the dependent $\operatorname{SDD}_{n_{3}}(t)$ and independent demand $\mathrm{SD}_{n_{3}}(t)$ for item $n_{3}$ in period $t$ are determined. $\operatorname{ID}_{n_{3}}(t)$ is the independent demand for item $n_{3}$ in period $t$ and $\mathrm{DD}_{n_{3}}(t)$ is the dependent demand for item $n_{3}$ in period $t$.

$$
\begin{aligned}
& \operatorname{SD}_{n_{3}}(t)=\mathrm{ID}_{n_{3}}(t)-\mathrm{UD}_{n_{3}}(t), \quad n_{3}=1, \ldots, N_{3}, t=1, \ldots, T \\
& \operatorname{SDD}_{n_{3}}(t)=\mathrm{DD}_{n_{3}}(t)-\mathrm{UDD}_{n_{3}}(t), \quad n_{3}=1, \ldots, N_{3}, t=1, \ldots, T
\end{aligned}
$$

The dependent demand $\mathrm{DD}_{n_{3}}(t)$ is determined by multiplying the BOM-factor with $\mathrm{TP}_{n_{2}}(t-L)$ with $L$ is the lead time of second stage of the supply chain.

$$
\mathrm{DD}_{n_{3}}(t)=\mathrm{BOM}_{n_{3}, n_{2}} \sum_{n_{2}} \mathrm{TP}_{n_{2}}(t-L), \quad n_{3}=1, \ldots, N_{3}, t=1, \ldots, T
$$


The backorder quantity for item $n_{3}$ in period $t \mathrm{BO}_{n_{3}}(t)$ is the summation of the unsatisfied dependent and independent demand for item $n_{3}$ in period $t-1$.

$$
\mathrm{BO}_{n_{3}}(t)=\mathrm{UDD}_{n_{3}}(t-1)+\mathrm{UD}_{n_{3}}(t-1), \quad n_{3}=1, \ldots, N_{3}, t=1, \ldots, T
$$

Finally, non-negativity constraints have to be considered.

$$
\begin{aligned}
& \mathrm{EI}_{n_{1}}(t), \mathrm{TD}_{n_{1}}(t), \mathrm{TR}_{n_{1}}(t), \mathrm{SM}_{n_{1}}(t), \mathrm{BM}_{n_{1}}(t), \mathrm{SD}_{n_{1}}(t), \mathrm{UD}_{n_{1}}(t), \mathrm{EI}_{n_{2}}(t), \\
& \mathrm{TD}_{n_{2}}(t), \mathrm{TR}_{n_{2}}(t), \mathrm{PP}_{n_{2}}(t), \mathrm{UD}_{n_{2}}(t), \mathrm{UDD}_{n_{2}}(t), \mathrm{SD}_{n_{2}}(t), \mathrm{SDD}_{n_{2}}(t), \mathrm{EI}_{n_{3}}(t), \\
& \mathrm{TD}_{n_{2}}(t), \mathrm{TR}_{n_{2}}(t), O_{n_{2}}(t), \mathrm{UD}_{n_{2}}(t), \mathrm{UDD}_{n_{2}}(t), \mathrm{SD}_{n_{2}}(t), \mathrm{SDD}_{n_{2}}(t) \geqslant 0
\end{aligned}
$$

\subsection{Backorders and safety stocks}

After solving the mathematical programming model that we discussed in the previous section, the planning horizon is shifted by one period after which the demand generator generates a new series of forecasts for the shifted horizon. The order releases within the frozen horizon determined in the previous solving round are not allowed to be changed, as these orders are assumed to be scheduled in a more detailed planning level or already taken in process. The supply chain planning model is solved again, but since a frozen horizon, fixed order releases and an update of the forecasts are taken into consideration, backorders may occur if the available inventories are no longer sufficient to satisfy the updated required quantities.

The planned backorder quantities after each solving round are stored. A large number of replications is necessary to draw valid conclusions on the empirical distribution of the backorders. Furthermore, the results of the first couple of runs have to be ignored, as the system has to reach a state that is independent of the initial conditions. The relation between the backorder quantities and the determination of safety stock levels will be explained in the following. Suppose that the safety stock levels were set beforehand equal to the maximum measured backorder quantities at all stages in the supply chain, a service level of $100 \%$ would have been achieved in the supply chain, given the generated forecasts of the demand process. Therefore, the last step of the approach is to set a target customer service level for the several items at the several stages. Based on these target customer service levels, safety stock levels can be determined for the items.

The service level for the most downstream stage (stage 1) in the supply chain is determined by Eq. (28) where $\beta_{n_{1}}$ is the fill rate for item $n_{1}$, i.e. the long-run fraction of independent demand $\operatorname{ID}_{n_{1}}(t)$ satisfied directly from shelf (without backordering).

$$
\beta_{n_{1}}=\sum_{t}\left(1-\frac{\mathrm{UD}_{n_{1}}(t)}{\mathrm{ID}_{n_{1}}(t)}\right), \quad n_{1}=1, \ldots, N_{1}
$$

For stages 2 and 3 of the supply chain, Eq. (29) determine the service level, as these stages face exogenous (independent) demand and endogenous (dependent) demand from the next (downstream) stage of the supply chain. 


$$
\beta_{n_{j}}=\sum_{t}\left(1-\frac{\mathrm{UD}_{n_{j}}(t)+\mathrm{UDD}_{n_{j}}(t)}{\mathrm{ID}_{n_{j}}(t)+\mathrm{DD}_{n_{j}}(t)}\right), \quad j=\{2,3\}, n_{j}=1, \ldots, N_{j}
$$

\section{Application of the approach}

The discussed approach has been implemented at Organon, a worldwide operating biopharmaceutical company with an annual turnover of more than 2.4 billion Euros. The company consists of more than 10 production sites and about 60 national distribution centres spread all over the world. Organon has more than 30 branded products in its portfolio and markets only prescription medicines for improving both the health and quality of human life.

Figure 4 shows a rough outline of one of Organon's tablet supply chains with the main production processes and stockpoints. Active ingredients form input to the tablets production process. Some additional materials may be needed for this production process. The packaging process blisters the tablets, packs the blistered tablets in cartons and instructions for use are added. Next, the finished products are shipped to more than 60 national warehouses (which are owned by Organon) spread all over the world. From these national warehouses, finished products are sold and distributed to customers like hospitals, pharmacists, and wholesalers.

This supply chain is planned and controlled by an APS, which was implemented a couple of years ago. The APS is a planning system that controls the supply chain by calculating high-level production plans for the several stages in the supply chain. The forecasts which are input to the planning problem are provided by the forecasting system which calculates statistical forecasts of the expected demand on SKU level based on historical demand information. Having implemented the Advanced Planning and Scheduling system, Organon was facing the question how to determine the safety stock parameters (which are input to the planning models) that cover (partially) demand uncertainties such that the entire supply chain is considered and total inventory holding costs are minimized given certain customer service levels.

In the following sections, we discuss the results of a project performed within Organon to determine the safety stock levels using the discussed approach. For confidentially reasons, the product names for which this approach has been implemented will not be mentioned. Further, the numbers do not reflect the real numbers, as they are divided by an arbitrary factor. With respect to the simulation experiment, the run length was set equal to 100 periods and the number of replications was five, following the approach proposed by Law and Kelton (2000). The average CPU time is about 2 mins. The supply chain planning model has been implemented in a standard Advanced Planning System that uses CPLEX as solver.

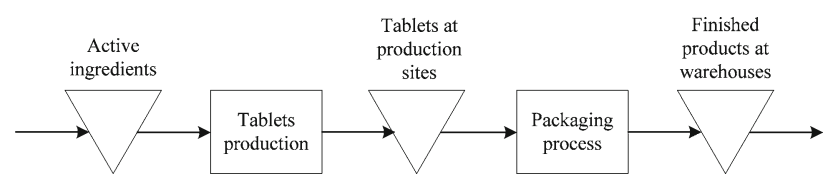

Fig. 4 A rough outline of the supply chain of Organon 


\subsection{The supply chain of product A}

Figure 5 shows the supply chain of product A. The most upstream stage in this supply chain is the active ingredient (AI 1). The tablet production is performed at two production sites of Organon, and therefore, active ingredients 1 (AI 1) are shipped to another production site (AI 2). After tablets production 2 (TB 2), the tablets are packaged and shipped to warehouses 1 till 11, which supply Organon's end customers. Production site 1 is supplying warehouses 12 till 26. Warehouses 27 till 34 are supplied by local subcontractors who get the active ingredients from Organon. Therefore, a direct link has been made between the stockpoints AI 1 and warehouses 27 till 34 .

We applied the proposed approach to this supply chain to determine the safety stocks levels of each item at each stockpoint. We note that safety stocks cannot be pooled, since the products in each warehouse are different due to the fact that they are country-specific. The lead times, batch sizes, bill-of materials structure and all other characteristics of this supply chain have been taken over from the supply chain planning model. The mathematical formulation of the mixed-integer programming model that is solved in a rolling horizon setting is the one discussed in Sect. 4.2.

Based on stored historical demand and forecasts data, we found that the normal distribution is statistically fitting the forecasts and sales data the best. A goodness-offit test has been used to find the suitable distribution that fits the best to the data. The result was that the average demand is time-independent, but forecast errors showed a strong correlation with the forecast age, i.e. the number of periods between the moment the forecast was made and the moment the demand is realized. The demand generator randomly generates a series of forecasts based on the parameters of the normally distributed demand $\left(\mu_{d, i}, \sigma_{d, i}(h)\right)$.

Fig. 5 The supply chain of product $\mathrm{A}$

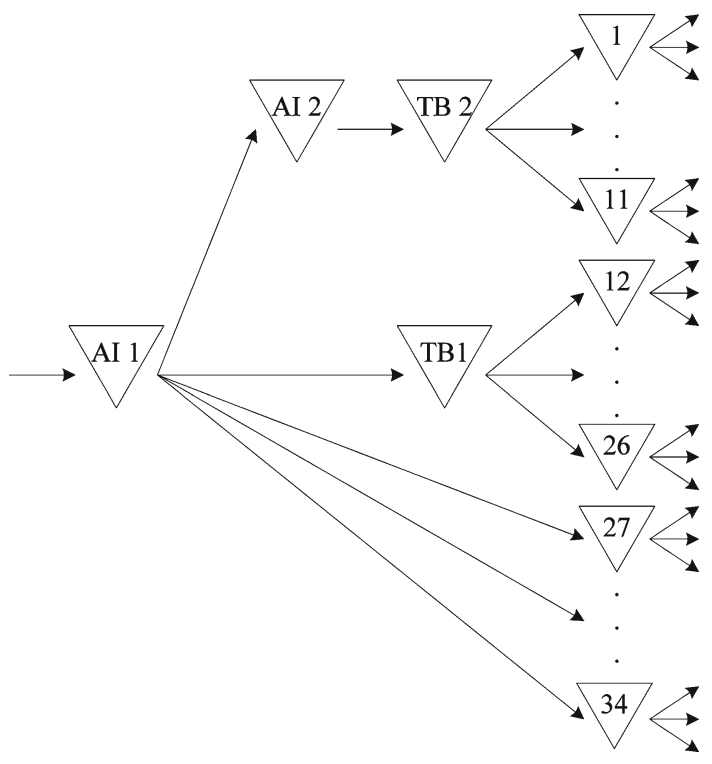




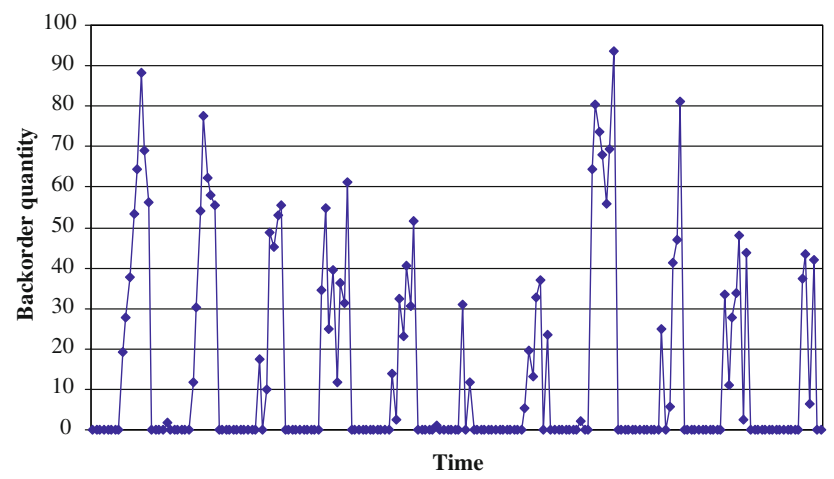

Fig. 6 An example of the backorders that result from a simulation study for a certain item

Table 1 Safety stock levels based on the proposed approach and the current safety stock levels

\begin{tabular}{lrl}
\hline & Current situation & Model suggestion \\
\hline National warehouses & 100 & 89 \\
Tablets stockpoint & 51 & 47 \\
Active ingredients & 49 & 21 \\
\hline
\end{tabular}

As discussed in the previous section, the outputs of the approach are series of planned backorders that are stored. Figure 6 shows the development of backorders of a certain item. As this figure shows, the backorder quantities are mostly equal to zero, which means that there is mostly enough inventories available to satisfy the required quantities. Whenever the required quantity (either dependent or independent demand) is not (fully) satisfied, a backorder is planned for the next period.

It is not possible to show here all results that we obtained from the implementation of the discussed approach. However, the results of our approach for this particular supply chain are presented in Table 1 . The second column of Table 1 shows the current safety stock levels and the third column shows the safety stock levels that result from our approach based on a target service level of $99 \%$. It was not our intention to decrease current safety stock quantities, but Table 1 shows that in this case, substantial savings may be achieved by implementing the approach. However, the comparison is not completely fair, as in the current situation also other types of uncertainties are covered.

\subsection{The supply chain of product B}

The supply chain of product B has also been used for the validation of the proposed approach. Figure 7 shows the supply chain of product B. Contrary to product A; two active ingredients (AI 1 and AI 2) are required for the tablet production. After the production process, the tablets are shipped to two packaging sites where the tablets are also stored (TB2 and TB3). After packaging of TB2, the products are shipped to 19 national warehouses all over the world, whereas TB3 is shipped to only one national 
Fig. 7 The supply chain of product B

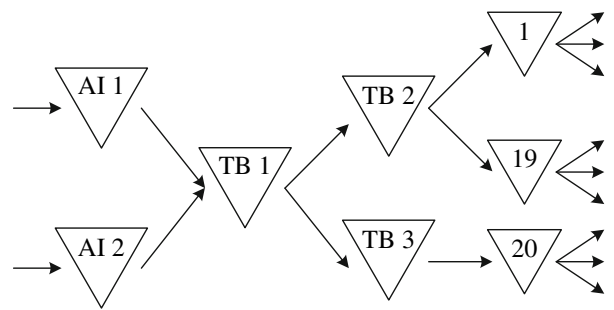

Table 2 Safety stock levels based on the proposed approach and the current safety stock levels

\begin{tabular}{lcc}
\hline & Current situation & Model suggestion \\
\hline National warehouses & 162 & 136 \\
Tablets stockpoint & 71 & 46 \\
Active ingredients & 29 & 22 \\
\hline
\end{tabular}

warehouse. The same approach has been applied for this supply chain to determine the safety stock levels. As we mentioned in the case of supply chain of product A, safety stocks can not be pooled due to the fact that each product is country-specific. Based on historical demand and forecasts data, the demand generator generates a series of forecasts that are input to the mathematical programming model that is presented in Sect. 4.2.

Table 2 presents the results of the proposed approach (to obtain a service level of 99\%) and the current safety stock levels. The results show that substantial savings can be made, but even more important, the approach turns out to give satisfying and reasonable results.

\section{Conclusions}

In this paper, we introduced an approach to determine safety stock levels in multi-item multi-stage inventory systems that face demand uncertainties. The problem of determining safety stock levels in a supply chain to meet certain predefined target customer service levels is based on a simulation study where the supply chain planning problem is solved in a rolling horizon setting. We assume that the supply chain is planned and controlled by a central authority that sets releases to the production system based on mathematical programming models. Combining the long run backorder quantities that result from the simulation study with predefined target customer service levels, the approach allows for determining safety stock levels in the supply chain.

The approach does not make any assumption about the demand process. Furthermore, all kinds of constraints can be included that are also considered in the supply chain planning model. The approach is based on two main assumptions. The first assumption is that the requirement process and replenishment decisions are completely independent from the safety stock levels. The second assumption is that all unsatisfied demand is backordered. As a form of validation, we discussed an application of the approach to two supply chains at Organon, a worldwide operating pharmaceutical 
company. The approach helped the company to determine the safety stocks that cover demand uncertainties.

A shortcoming of our approach is that we assume that any upstream unavailability of stock leads to an order delay at the next stage, which affects the performance of the inventory system. This is not necessarily what happens in practice. A short study that we performed showed that usually protection against a shortage is not only achieved through the use of safety stocks, but also by using the slack in the lead times or by reprioritizing the orders such that a higher customer service level is achieved than initially planned. This effect can be compensated by setting the target customer service level lower than the 'real' target customer service level and this could be an object of further study.

Acknowledgments The authors would like to extend their word of thanks to Organon, especially to John Koelink and Joop Wijdeven for initiating and supporting this project. Further, we would like to thank two anonymous referees for their valuable and constructive comments that helped to improve the clarity of this paper.

\section{References}

Billington PJ, McClain JO, Thomas LJ (1983) Mathematical programming approaches to capacityconstrained MRP systems: review, formulation and problem reduction. Manage Sci 29:1126-1141

Callarman TE, Mabert VA (1978) Using material requirements planning with demand uncertainty. In: Proceedings of the 9th annual midwest AIDS conference. pp 151-155

Callarman TE, Hamrin RS (1984) A comparison of dynamic lot sizing rules for use in a single stage MRP system with demand uncertainty. Int J Oper Prod Manage 4(2):39-49

Clark AJ, and Scarf H (1960) Optimal policies for a multi-echelon inventory problem. Manage Sci 6: 475-490

De Bodt MA, Van Wassenhove LN (1983) Lot sizes and safety stocks in MRP: a case study. Prod Inventory Manage 24(1):1-16

De Kok AG, Fransoo JC (2003) Planning supply chain operations: definition and comparison of planning concepts. In: De Kok AG, Graves SC (eds) Design and analysis of supply chains: design, coordination and operation (Handbooks in Operations Research and Management Science, Volume 11). North Holland, Amsterdam, pp 597-675

Diks EB, De Kok AG, Lagodimos AG (1996) Multi-echelon systems: a service measure perspective. Euro J Oper Res 95:241-263

Eilon S, Elmaleh J (1968) An evaluation of alternative inventory control policies. Int J Prod Res 7(1):3-14

Graves SC, Willems SP (2000) Optimizing strategic safety stock placement in supply chains. Manu Serv Oper Manage 2(1):68-83

Heath DC, Jackson PL (1994) Modeling the evolution of demand forecasts with application to safety stock analysis in production/distribution systems. IIE Trans 26(3):17-30

Inderfurth K (1991) Safety stock optimization in multi-stage inventory systems. Int J Prod Econ 24:103-113

Inderfurth K, Minner S (1998) Safety stocks in multi-stage inventory systems under different service measures. Eur J Oper Res 106:57-73

Kleijnen JPC, Wan J (2006) Optimization of simulated inventory systems: OpQuest and alternatives. Tilburg University: CentER Discussion Paper, no. 2006-75

Kohler-Gudum CK, De Kok AG (2002) A safety stock adjustment procedure to enable target service levels in simulation of generic inventory systems. Technische Universiteit Eindhoven: BETA Working Paper 71

Law AM, Kelton WD (2000) Simulation modelling and analysis, 3rd edn. McGraw-Hill, New York

Minner S (1997) Dynamic programming algorithms for multi-stage safety stock optimization. OR Spektrum 19:261-271

Silver EA, Pyke DF, Peterson R (1998) Inventory management and production scheduling. Wiley, New York 
Spitter JM, Hurkens CAJ, De Kok AG, Negenman EG, Lenstra JK (2005) Linear programming models with planned lead times. Eur J Oper Res 163:706-720

Stadtler H (2003) Multi-level lot sizing with setup times and multiple constrained resources: internally rolling schedules with lot-sizing windows. Oper Res 51(3):487-502

Stadtler H, Kilger C (2005) Supply chain management and advanced planning, 3rd edn. Springer, Berlin

Tempelmeier H, Derstroff M (1996) A Lagrangean-based heuristic for dynamic multi-level multi-item constrained lotsizing with setup times. Manage Sci 42(5):738-757

Van Houtum GJ, Inderfurth K, Zijm WHJ (1996) Materials coordination in stochastic multi-echelon system. Eur J Oper Res 95:1-23

Wemmerlöv U, Whybark DC (1984) Lot-sizing under uncertainty in a rolling schedule environment. Int J Prod Res 22(3):467-484

Whybark DC, Williams JG (1976) Material Requirements Planning under uncertainty. Decis Sci 8(4)

Wijngaard J, Wortmann JC (1985) MRP and inventories. Eur J Oper Res 20:281-293 